\title{
Business travel - the social practices surrounding meetings
}

\author{
Glenn Lyons ${ }^{a}$ \\ ${ }^{a}$ Centre for Transport \& Society, Faculty of Environment and Technology, University of the \\ West of England, Frenchay Campus, Bristol, BS16 1QY. United Kingdom. \\ Tel +44 1173283219 Fax +44 1173283899 Email Glenn.Lyons@uwe.ac.uk
}

\begin{abstract}
A considerable amount of travel domestically and internationally is undertaken by 'briefcase travellers' in the pursuit of business meetings. Such business travel is deemed costly to the economy. This paper examines the potential factors at work in the social construction of meetings and their associated travel. What are the different motivations and expectations in attending a meeting? What are the actual consequences (positive and negative) in attending? How can the organisation of a meeting impact upon the wider organisation of activity in time and space of the individuals involved? How does the process of meeting attendance, including travel, unfold? How might ICTs impact on the social practices associated with meetings? The paper offers a critical assessment of such issues that may underlie and influence the nature and extent of business travel. It goes on to define the notion of excess briefcase travel as a means to frame the challenge for policymakers and employers and employees in potentially reducing such travel and the associated research challenge to establish empirical understandings. The paper examines literatures from transport studies as well as other territories of social science including mobilities research.
\end{abstract}

Keywords: Business travel, briefcase travel, meetings, excess travel 


\section{Business travel - the social practices surrounding meetings}

\section{$1 \quad$ Introduction}

As currently judged within transport economics, business travel accounts for a major part of the economic value of time invested in travel overall. It therefore seems remarkable how little attention within transport studies had been paid to developing an understanding of the composition of business travel and what gives rise to such travel. An improved understanding would help inform how this might be changing or could be changed in future. Both transport policy and business practices within employers face the challenges of addressing a radically changed economic landscape, pressing environmental issues and managing the demand for travel. They also face the opportunity of the information age in terms of new ways of transacting that may (or may not) hold the promise of reducing or reshaping the amount of business travel that takes place. This paper seeks to draw upon what literature does exist both within transport studies and more broadly in other fields such as that of mobilities in the social sciences in order to explore attitudes, behaviours and influencing factors regarding meetings and the associated business travel. The aim is to reveal the extent of current understanding and explore what prospects might exist for changing levels of business travel.

As at $2011,3 \%$ of all domestic trips made in Great Britain were for business ${ }^{1}$ (i.e. travel during the course of work) compared to $15 \%$ for commuting (DfT, 2012). The figures for the US (for 2001) are remarkably consistent with work related business accounting for $3 \%$ of all person trips and trips to/from work accounting for $16 \%$ of all person trips (Hu and Reuscher, 2001). Meanwhile, international business travel has grown in tandem with a globally networking society - Haynes (2010) points to there being more international business travel by air today than the total amount of international air travel in 1980.

\footnotetext{
${ }^{1}$ Defined in the National Travel Survey as "personal trips in course of work, including a trip in course of work back to work. This includes all work trips by people with no usual place of work (e.g. site workers) and those who work at or from home".
} 
In terms of the cost to the economy of business trips (as interpreted in the UK), the value of working time per person associated with a journey depends upon the travel mode (based on the average wage rate of individuals using a given mode). Hence the 'cost' of a business trip by car (as the driver) lasting one hour is assumed to be $£ 26$ per individual in terms of time lost to economically productive use outside of travel. The corresponding figure for a rail passenger is $£ 37$. Meanwhile the value of a one hour commute (based on an equity value of willingness to pay of the individual) in terms of time 'cost' is taken to be $£ 5$ (DfT, 2011). Once such values are accounted for it becomes clear that, in spite of their small share of overall (domestic) trips, in terms of assumed economic impacts of travel, business trips account for a substantial proportion of total travel. Mackie et al (2003) note that "[f]or proposed road schemes ... although business travel by car only accounts for around one sixth of all traffic, it accounts for about half of the assumed 'costs' of travel time".

From a transport policy perspective we know remarkably little about business travel in terms of understanding its determinants and potentially influencing its overall impact on levels of mobility. Indeed, literatures beyond those of transport studies attest to a wider paucity of insight (e.g. Gustafson, 2006; Faulconbridge and Beaverstock, 2008; Beaverstock et al, 2010; and Haynes, 2010). This said, it is within such literatures that some researchers are seeking to address the issue (variously focusing on international as opposed to domestic travel (e.g. Faulconbridge and Beaverstock, 2008; Beaverstock et al, 2010; and Haynes, 2010), overnight business trips as opposed to within-day trips (e.g. Gustafson, 2006) and business travel by air (e.g. McNeill,2009; Haynes, 2010; and Denstadli et al, 2012)).

The paper uses a series of questions to frame an examination of the issues and considerations that are contributing or could contribute to a developing understanding of business travel - or more specifically that travel which derives from the participation in face to face meetings. 
In order to move to a position of considering face to face meetings and their associated travel, it is first necessary to recognise the breadth of coverage of travel purposes that can be reflected in the rather ambiguous term 'business travel'.

\subsection{What is the makeup of business travel and its destination activities?}

National travel surveys are a key source when painting a picture of the composition of (domestic) travel according to journey purposes. However, in spite of their authoritative nature in relation to substantial sample sizes and thus representativeness, they report in a remarkably superficial manner in terms of disaggregated insight into the nature and heterogeneity of travel and travel purpose. In Great Britain's National Travel Survey, in common with other such surveys, a key element is a self-completion 7-day travel record (Anderson et al, 2009). In terms of recording journey purpose, participants are instructed as follows: "What was the purpose of your journey? Please give a simple description such as 'go to work', 'take children to school' or 'go home', If you went shopping please note whether it was 'food shopping' or 'other shopping' ". These data are then processed into 20 different trip categories, one of which is 'in course of work'.

Thus, all we know in relation to business travel from such surveys is what proportion of all trips the rather coarse category 'in the course of work' represents. Similar limitations apply to records of international travel. It can be tempting to characterise all 'business travel' from the perspective of the knowledge worker. However, Mackie et al (2003) remind us that "[t]here is clearly a category of employers' business travel in which, broadly speaking, the work being done during employers' business time actually consists of travelling: this applies, for example to service engineers, delivery people, public transport drivers, lorry drivers etc." Meanwhile these authors have coined the phrase 'briefcase traveller' to reflect employees 
travelling in the course of business. This can be taken to refer to that travel associated with face to face meetings.

There are a number of considerations that would shed more light on the makeup of business travel in relation to an interest, eventually, in addressing travel demand and patterns of travel associated with business activities.

The extent to which the activity at the destination is location dependent

Some travel in the course of work has destinations that are spatially fixed: the service engineer's visit to a given premises to carry out work; the delivery of a parcel to a specified address; a site visit to inspect or discuss specific facilities. Meanwhile briefcase travel may have few or no constraints on destination location in terms of the destination activity itself other than the provision of suitable facilities such as a meeting room. In such cases a choice of destination is likely to exist (especially for domestic business travel). The question then becomes one of what factors determine the choice outcome. Destination may be dictated by habit or tradition (e.g. meeting at company HQ), the location of the activity host or by a 'boundedly rational' (Todd, 2007) optimal location to suit all those involved in (travelling to) the activity. The level of seniority of individuals involved or spatio-temporal constraints faced by specific individuals may weight such location choice (Urry, 2003). Consideration of collective carbon footprint may also now feature.

The nature of the activity at the destination in terms of the importance of co-presence

Allied to the issue of location dependence is the question of whether two or more individuals need to be co-present in order that the purpose(s) of the activity at the travel destination are achieved? This question is particularly pertinent where the activity concerns information exchange and where it may appear that the information could have electronically 
transcended the distance between the individuals involved rather than the individuals having to travel to be co-present.

Co-presence offers the prospect of a multi-sensory experience of encounter and exchange - while the exchange of facts and figures, diagrams and the like could be done remotely, matters of eye contact, body language and indeed smell can strongly colour the proceedings and outcomes of an activity. Arnfalk and Kogg (2003) and Denstadli et al (2012) review media richness theory which contends that the medium of communication is determined by the nature of information to be communicated - more complex information requiring higher 'bandwidth' media (notably face to face) and straightforward information requiring only a low-density medium (e.g. email). An important distinction must be made between the transmission of codifiable information with 'stable meaning' and of 'complex tacit knowledge' (Storper and Venables, 2004). Face to face lends itself strongly to the latter.

There is a danger of 'functional thinking' (Geels and Smid, 2000) in understanding the purpose of face to face communication. It will often reach beyond sharing knowledge and views on explicit topics of business relevance to the formation of trust and building of human relationships. As Storpher and Venables (2004) note, "[h]umans are very effective at sensing non-verbal messages from one another particularly about emotions, cooperation, and trustworthiness"; likewise Urry (2003) remarks that "[e]ye contact enables and stabilizes intimacy and trust".

It can also be the case that co-presence provides a contribution to social capital. Jain and Lyons (2008) discuss the notion of the gift of travel time which can in part symbolise the gesture of importance of a social encounter implied by an individual being prepared to give their time in order to travel and achieve co-presence (in contrast to the fast and efficient alternative of electronic communication which "paradoxically, can be so efficient it that it destroys the value of the message" (Storper and Venables, 2004)). Urry (2003) more broadly explores what he calls 'meetingness' and, in the context of an increasingly networked society of the information age, the need for periodic face to face meetings (social or business) to address obligations as well as to sustain networks of weak ties. Faulconbridge et al (2009) in 
their empirical analysis of professional services firms reveal an intermittence of face to face meetings that are "the opportunity to establish, consolidate and reconfirm relationships which minimise the future need for travel".

The duration of the activity set against the duration of the associated travel

Schwanen and Dijst (2002) have examined the relationship between activity duration and travel time considering the notion that individuals balance the two. Based on empirical evidence from the Dutch national travel survey - for commuting - it was found that there was considerable variation around an average travel-time ratio value. Travel-time ratio is defined as "the ratio between travel time and the sum of travel time and activity duration". The average value of the ratio they calculated for a car driver was 0.111 and for a train passenger was 0.222 . It would be instructive to have similar values available for business trips. On the one hand one can envisage the ratios being (a lot) higher for single day business trips. Meanwhile for business activities lasting more than a day (e.g. a 2-3 day workshop or conference) the ratio could be (a lot) lower. It may be assumed that individuals would have a notional 'ratio threshold' above which they could not justify engagement in the business activity. On the other hand, achieving a positive utility from the travel time itself in terms of travel time use (Mokhtarian and Salomon, 2001; Lyons and Urry, 2005) could push any such threshold higher. There would also be the wider implications to consider of how much time is devoted in sum to business travel and how this affects availability for work and for family (Bergman and Gustafsen, 2008).

It is not clear to what extent those organising meetings or individuals attending meetings consciously consider the travel 'cost' versus the benefits of activity attendance or whether other factors are overriding and meeting attendees are relied upon to manage the travel time ratios they face. 
Another means of offsetting the travel 'cost' of a business activity and improving the traveltime ratio is to cluster more than one business activity in (close) spatial and/or temporal proximity. One business activity acts as a spatial and/or temporal 'anchor' around which other activities can be arranged. For example McNeill (2009), in examining the airport hotel as a business space, points to the high proportion of international business trips by air that "involve a sequence of meetings with different clients or contacts". Two considerations arise. Firstly there is the ability of an individual to dictate the timing and/or location of a further activity to correspond to the anchor - this will depend upon their level of influence/importance associated with the further activity's scheduling and their preparedness to optimise their scheduling. (Urry (2003) refers to 'complex inequalities of power' in the convening of meetings.) Secondly, a lower level of 'necessity for attendance' threshold for any given activity in the prospective cluster may arise by virtue of the shared travel time cost between the activities.

\subsection{To what extent are business meetings 'necessary' as events and from the perspective of each individual attending?}

Meeting attendance as a whole (including 'office' meetings as well as intra-organisational and inter-organisational meetings drawing on attendees from multiple locations) reflects a substantial investment of time for some workers (Arnfalk and Kogg, 2003). Romano and Nunamaker (2001) point to studies of managers and knowledge workers which "reveal that they spend between $25 \%-80 \%$ of their time in meetings". A central issue determining both whether a business meeting takes place and who is in attendance (both of which fundamentally affect the amount of associated travel unless it is an in-house meeting) is the extent to which conscious consideration is given to whether or not the need for the activity going ahead or any one individual's participation can be justified. It would seem more than a 
co-incidence that meetings are the source of popular derision in terms of their effectiveness, as noted by the following quotes:

- "Meetings are indispensable when you don't want to do anything" (John Kenneth Galbraith)

- $\quad$ "The least productive people are usually the ones who are most in favor of holding meetings" (Thomas Sowell)

- "A committee is a group of the unwilling chosen from the unfit, to do the unnecessary" (Unknown author)

These would suggest some sense that greater efficiencies should indeed be possible (in terms of meetings that are worthwhile and for whom it is worthwhile to attend) where time investment in meetings is concerned. Romano and Nunamaker's examination of meeting data for corporate America suggested a prevailing view that, because meetings can suffer from a number of inefficiencies in their organisation and conduct, meetings are generalised to be unproductive and wasteful (Romano and Nunamaker, 2001).

While a degree of consideration is typically given to convening a meeting and its attendance, it can also be the case that such consideration falls 'victim' to choice mechanisms that are also being recognised in relation to travel (Chorus et at, 2006; Lyons et al, 2008a) - in particular habitual behaviour and satisficing behaviour. In terms of habit, some meetings are 'routine' and are convened and put into people's diaries almost without question regarding the appropriateness of their frequency, location or indeed need. Consideration of whether or not a meeting should be arranged and whether an individual should attend may be partial in terms of weighing up the pros and cons. It only needs the case for proceeding to be 'satisfactory' for the affirmative decision to be made. Ivancevich et al (2010) bemoan the lack of managerial attention given to business travel and the fact that "despite the significant economic expenditures of business travel and its role in globalization and nurturing business transactions there is no available framework that focuses on the stresses, strains and outcomes associated with it". 
One can conceive of a number of considerations that are in the mind of the individual when making the decision on whether or not to attend a meeting (involving travel). This is reflective of Arfalk and Kogg (2003) who acknowledge (in their examination of the notion of an 'optimal meeting') "the complex range of aspects that influence the costs and benefits that relate to a meeting". It should be noted of course that the extent to which an individual has discretion over whether or not to attend (or convene) a meeting will vary - such decisions may in fact be in the hands of their superiors. An illustrative rather than exhaustive set of such considerations is shown in Table 1. The inclusion of ticks in the Table is in some cases speculative but reflects an important observation that some considerations may be judged in opposite ways by different individuals or by the same individual in relation to different situations.

Table 1. Individuals' possible considerations on the merits of meeting attendance

\begin{tabular}{lcc}
\hline Consideration & $\begin{array}{c}\text { Potential } \\
\text { motivation / }\end{array}$ & disincentive \\
& benefit & / disbenefit \\
& $\checkmark$ & $\checkmark$ \\
\hline Business norm & $\checkmark$ & $\checkmark$ \\
Time away from 'the office' & $\checkmark$ & $\checkmark$ \\
Time away from home & $\checkmark$ & \\
Information sharing - knowledge exchange & $\checkmark$ & \\
Influencing decisions & $\checkmark$ & $\checkmark$ \\
Status and recognition & $\checkmark$ & $\checkmark$ \\
Networking & $\checkmark$ & $\checkmark$ \\
Sociability & $\checkmark$ & $\checkmark$ \\
Experiencing new places & & \\
Monetary cost & & \\
\end{tabular}


An individual may consciously or perhaps unconsciously sense an expectation that they attend a meeting because it is a business norm to do so - this may be something that is taken as an overriding justification for attendance. On the other hand it may in some cases be recognised as an equally overriding justification for not attending if the individual senses this is an underlying motivation for the meeting as a whole with prospects for inefficient use of time by attending.

Time away from the office in order to travel to/from and attend a meeting may be seen as an opportunity cost ${ }^{2}$. Certainly in terms of economic appraisal of business travel in the UK it is judged as such. Ivancevich et al (2010) identify pre-, during- and post-trip stressors resulting from taking time away from the office - delegating projects and accumulated work. However, for some individuals there may be no opportunity cost at all but instead an opportunity benefit in that travelling to/from the meeting offers the best alternative in terms of their productive time use - time, for example, to concentrate that is not afforded in the office.

Boundaries between work time and non-work time for some in the knowledge economy / information age can become increasingly blurred. Meeting attendance can result in early starts or late arrivals home on a given day or indeed in days and nights away from home. This time away from home may for some be an attraction of meeting attendance. It would be presumed that for a greater proportion of individuals time away from home is seen as a personal opportunity cost associated with meeting attendance that would weigh in favour of not attending the meeting (and there are distinct gender differences here (Bergman and Gustafson, 2008)). Ivancevich et al (2010) identify stressors concerning making home arrangements and unmet family responsibilities and point to fatigue, frustration and anxiety being caused by business trips due to worry about effects at home (or at work). In an examination of gender and family obligations in relation to business travel, Gustafson (2006) points to the phenomenon of 'intermittent husband syndrome' with potential stress problems for the business traveller as well as marital problems and behavioural problems in children.

\footnotetext{
${ }^{2}$ Defined by the Collins English Dictionary as "cost in terms of the best alternatives foregone".
} 
In terms of the meeting itself then perhaps two of the most tangible reasons for attendance concern information sharing and the influencing decisions. While the individual (or their employer) may not consider a (co-present) meeting was necessary in order that information be shared and decisions reached, given that the meeting is to proceed it becomes necessary to participate. Allied to business norms and with the earlier reference to the gift of travel time, status and recognition can be a significant consideration. This may be equated to presenteeism in that whether or not the individual makes a worthy contribution to the meeting they share in its value and significance by association (Urry, 2003). Three further considerations relate to indirect aspects of a convened meeting. Co-presence affords the opportunity for networking with others (both already known and new acquaintances) in relation to matters not necessarily concerned directly with the purposes of the meeting itself. Conceivably some individuals see this as a disincentive because of their own confidence in engaging though in the main this would be judged as a motivation for attendance and in some cases even a principal motivation for doing so. Closely related to networking is sociability in that individuals may thrive (or not) on the overspilling of business into pleasure in relation to shared time with colleagues and acquaintances that may be before, during or after the formal business of the meeting itself. The geographic location may form part of the associated sociability allied to the opportunity to experience new places (Urry (2003) refers to 'face-to-place' co-presence). Indeed, in some cases (partly because of opportunities to tag non-work social activities to a meeting) the location of the meeting can become the dominant factor in an individual being motivated to attend (or discouraged from attending) a meeting with the goals of the meeting itself and associated opportunity costs deemed to be secondary. Lastly there is typically a monetary cost, albeit borne by the employer, of meeting attendance that may bring into sharper relief overall the conscious consideration of the pros and cons of meeting attendance (for some specific figures on costs see Romano and Nunamaker (2001)). 
There will also be benefits and disbenefits that are not consciously considered or anticipated in the (cumulative) attendance of meetings such as serendipitous encounter and physiological effects (Ivancevich et al, 2010) respectively.

If we are to better understand how social and technological change may or could (with appropriate supporting policy interventions) affect the propensity for meetings to be convened and be attended in the future then further empirical insights are needed into the considerations above (and others that may also come to light). There is a need not only to understand the relative influence that such considerations have in the decisions to attend (and indeed convene) meetings but also to assess the degree to which such considerations can be influenced in the interests of reducing the overall need for motorised mobility associated with business meetings.

In terms of empirical evidence gathering it would be insightful not only to attempt to gather from individuals, in relation to specific meetings, their decisions on whether or not to attend and the considerations weighed up in doing so, but also to retrospectively assess the outcome of their decisions. In other words, having attended a meeting, to what extent did different considerations play their part (as expected or not) in determining the overall degree to which attendance at the meeting was beneficial? Follow-on questions would then be: to what extent do individuals ordinarily reflect upon the efficacy of meeting attendance?; and to what extent is there a learning process at work such that past behaviour is influencing subsequent decisions regarding meeting attendance? The paper returns to this later in Section 3.

\subsection{What are the consequences of meeting attendance for the individual in terms of their use of time?}

Assuming an individual has committed to attendance at a business meeting then this will clearly impact upon their work schedule for the given day or indeed week. The time devoted to the meeting itself might be assumed to be lost from the available time that day or week for 
other tasks and hence the prospect of opportunity cost exists (as noted earlier). This said, for longer business meetings - especially conferences over several days - it is not a foregone conclusion that the individual will be fully engaged throughout in the proceedings of the meeting. Such engagement may be punctuated by periods of 'time out' that are used for other purposes (e.g. working in one's hotel room). Indeed Middleton (2008) in her critical examination of the Blackberry phenomenon highlights how mobile technologies are allowing engagement in work activities "in locations and at times that were previously 'off limits".

If it is taken as given that the individual has suitably judged the matter of opportunity cost in terms of the time devoted to participating in the business activity itself then attention turns to the potential opportunity cost associated with the travel time devoted to travelling to/from the meeting location. Current UK transport economic appraisal reasons that there is indeed an opportunity cost associated with the travel in that an individual would prefer to use the travel time in some other more productive way if that time could be saved. However, such reasoning may be questioned in terms of how time and indeed working environment relate to productivity in the knowledge economy.

It may be assumed that many individuals involved in attending meetings are knowledge workers in some capacity. Holley et al (2008) discuss how the makeup of economic activity is changing with a move towards a greater proportion of knowledge work and suggest that "[a]s part of this change in the organization of work, there is now a reduced (and reducing) dominance of the clock-controlled industrialized time and a resurgence of the pre-industrial task-oriented concept of time." In other words it becomes difficult to judge objectively what opportunity costs are associated with the clock time set aside for travelling to/from and participating in a business meeting. An individual's productivity in the knowledge economy is more likely to be related to the outputs and outcomes of a task being completed than the Fordist metric of hours worked.

Recent research is revealing how travel time can be fruitfully used both for work and non-work activities (e.g. Lyons and Urry, 2005; Watts, 2008; Lyons et al, 2012). Table 2 
shows how individuals travelling for business ${ }^{3}$ in a large scale survey of rail passengers in Great Britain (Lyons et al, 2007) have used their travel time and how this has been judged in relation to a loose notion of productivity.

It is notable that $13 \%$ of business travellers indicate that they spent most of their time on the train working/studying and that they made very worthwhile use of their time ( $42 \%$ of the $31 \%$ of business travellers who spent most time working/studying). This suggests that for a substantial minority of individuals travelling to/from their business engagements the travel time may be a gift rather than a burden in terms of work activity. The following extract from a participant in previous qualitative research I have undertaken with colleagues illustrates this: "going up [on the train] was good because I was going for a meeting about the work magazine, I'm on the committee so it actually gave me two hours to read the magazine - the latest edition - so I could actually be ready for the meeting because I've always been too busy at work to find the two hours to sit and read a magazine."

What Table 2 also highlights is the challenge of determining 'ownership' of time in the knowledge economy. Economic appraisal assumes that travel during the course of work is time 'owned' by the employer. However, in a task-oriented approach to time then ownership of time is defined by what an individual is doing not by when or where they do it. Thus a quarter of rail business travellers use their travel time mainly for reading for leisure and indeed nearly 1 in 4 of these consider they make very worthwhile use of their time (see Table 2). Considered narrowly it may be assumed that this is an opportunity cost in economic terms but such individuals may just as likely be working at other times which would traditionally be deemed outside of the working day (see Bergman and Gustafson's examination of availability (2008)).

\footnotetext{
${ }^{3}$ Business travellers are identified in the National Rail Passenger Survey as 'On company business (or own if self employed)'. It cannot therefore be certain what percentage of these are travelling in relation to face to face meetings though the proportion is likely to be high.
} 
Table 2. Specific activities upon which business travellers spent most or some of their journey time (percentage of business travellers) and the corresponding assessment (percentage of relevant respondents) of the journey time use by those spending most time on a given activity (reproduced from Lyons et al, 2008b)

\begin{tabular}{|c|c|c|c|c|c|}
\hline Activity & 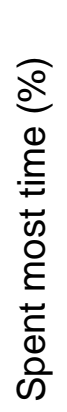 & 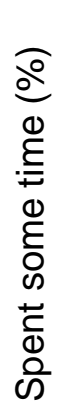 & 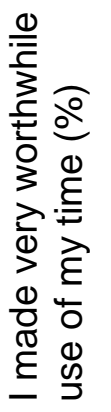 & 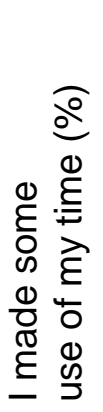 & 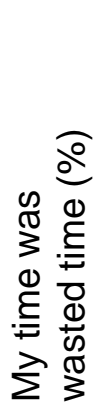 \\
\hline Working/studying & 31 & 51 & 42 & 54 & 2 \\
\hline Reading for leisure & 25 & 47 & 23 & 63 & 12 \\
\hline Window gazing/people watching & 13 & 53 & 12 & 58 & 28 \\
\hline Talking to other passengers & 5 & 13 & 24 & 56 & 19 \\
\hline Sleeping/snoozing & 3 & 13 & 15 & 57 & 27 \\
\hline Text messages/phone calls - work & 2 & 22 & 39 & 58 & 2 \\
\hline Text messages/phone calls - personal & 1 & 15 & 26 & 50 & 12 \\
\hline Eating/drinking & 1 & 21 & 19 & 80 & 1 \\
\hline \multicolumn{6}{|c|}{$\begin{array}{l}\text { Note: not all activities offered in the survey question are included in the Table - } \\
\text { only those that were selected by at least } 10 \% \text { of respondents for either most time }\end{array}$} \\
\hline
\end{tabular}

Consequences of meeting attendance in terms of travelling are also influenced by the choice of travel mode and the level of service offered by that mode (or combination of modes). A greater array of activity types can be engaged in as a rail passenger than as a car driver though assignment of tasks may mean an individual is able to gain as much benefit from a car journey as from a train journey. Stradling (2004) refers to three types of effort expended when making a journey: physical effort ("walking, waiting, carrying, escorting and 
maintaining body posture"); cognitive effort ("information gathering and processing for route planning, navigation, progress monitoring and error correction"); and affective effort ("the emotional energy expended on a journey in dealing with uncertainty about safe and comfortable travel and timely arrival at intermediate and final destinations"). Such effort, as well as contributing to stressors associated with business travel (Ivancevich, 2010) is likely to detract to varying degrees from an individual's ability to fully engage in worthwhile time use.

In summary, it remains unclear to what extent the travel component of attending a meeting detracts from (or indeed contributes to) an individual's overall productivity and wellbeing. It seems reasonable, however, to suggest that many individuals have, in principle, some control over this. It appears in the case of rail travel for example that the vast majority of business travellers are provided with an office on the move - $86 \%$ or respondents in the National Rail Passengers Survey in Great Britain answered 'yes' to the question 'in terms of your paid employment is there some work that could easily be undertaken on the train?' (Lyons et al, 2008b). Opportunities for greater flexibility of and control over time use are potentially offered through the growing prevalence of information and communications technologies (ICTs) in our working and personal lives. The proportion of business rail travellers who consider their time spent very worthwhile has increased by $20 \%$ between 2004 and 2010 (Lyons et al, 2012).

\subsection{In what ways and to what extent can ICTs play a part in reshaping business activity and travel?}

ICTs have become an increasingly pervasive feature of many people's working lives, especially for those in the knowledge economy: communicating by email and (mobile) phone; and sharing, exchanging and jointly working on documents. Inevitably therefore ICTS 
are likely to have potential roles in relation to business meetings and related travel. Two roles in particular stand out: substitution; and multitasking.

\section{Substitution}

It has long been assumed that it should be possible, in the case of a meeting largely or entirely concerned with information exchange, to let the information do the travelling rather than require travel by the individuals in order that they be co-present for the exchange. Teleconferencing technologies have been under development for many years. In spite of the rapid advances they continue to see (Denstadli et al, 2012) and the prospects they may hold for influencing the extent of business travel and its demands on environmental and human resource, research investigation remains sparse (Cairns et al, 2004). As Denstadli et al (2012) note, "[d]espite a growing acceptance of VC [videoconferencing] in modern organizations, empirical studies of its implementation and use are rare - particularly studies that compare VC to FTF [face to face] meetings".

It is notable that while videoconferencing is still evolving, for a number of people either through experience or perception it falls short of offering the appeal of the face to face communication channel (Denstadli et al, 2012; Haynes, 2010). Evidence from Sweden (Arnfalk and Kogg, 2003) suggests a preference for audioconferencing in terms of availability, ease of use and reliability. Email can also be considered a potential substitute for a face to face meeting. This points to the observation that substitution for a face to face meeting does not necessarily have to attempt to replicate the experience of a face to face meeting (as might be assumed in the case of videoconferencing). Substitution really concerns the (primary) purpose of a meeting rather than the means to that end. Email offers an asynchronous form of communication that for many is appealing in part because it can be accommodated around other tasks and/or because it allows more thoughtful and measured exchanges of information and opinion (Loch et al, 2003). 
High-end videoconferencing is coming closer to enabling eye contact and body language to play their part in meetings with improved audio-visual standards (see Figure 1). In terms of availability, web cams and products and services such as Skype ${ }^{4}$ are broadening the prospects of videoconferencing take-up, particularly if bandwidth and quality improvements continue.

According to media richness theory (referred to earlier), teleconferencing mechanisms may in time secure greater overlaps of capability with face to face meetings. As well as advances in capability and usability, this may arise in part due to what is known as channel expansion theory - the effectiveness of the medium expanding as users learn how to use it better and as communication through the medium becomes more intuitive (Arnfalk and Kogg, 2003).

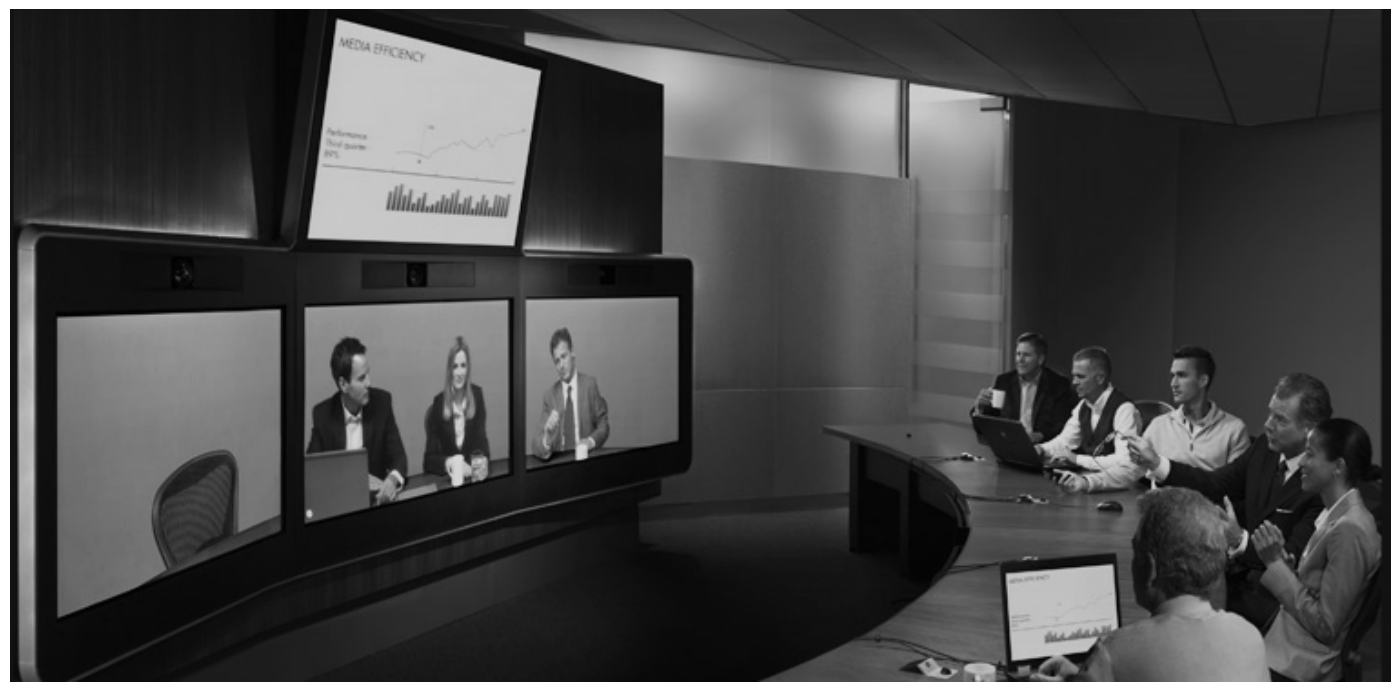

\section{Figure 1. HP Visual Collaboration Studio ${ }^{5}$}

The discussion above of substitution relates (implicitly) to substitution for a physical meeting taking place. However, substitution can also apply at the level of an individual. So, for instance, meetings can predominantly concern a group of individuals coming together but

\footnotetext{
${ }_{5}^{4}$ http://www.skype.com

$5 \frac{\text { Image from http://www.telepresenceoptions.com/studio-wide.jpg }}{\text { Imw }}$
} 
with provision for others to join through teleconference for all or part of the meeting. Indeed such substitution in some cases is symbolic of the times we live in, in the face of climate change: examples are now increasingly commonplace of leading figures choosing to deliver keynote addresses to conferences through a video link for reasons of time pressures in being unable to attend the event in person and/or of limiting the carbon footprint of their participation.

Contemplation of substitution implies replacement. However, commentators seem broadly agreed based on what evidence does exist (and broader inferences concerning human behaviour and networking) that face to face and virtual meetings will co-exist in a cooperative manner within business practices (Faulconbridge et al, 2009; Haynes, 2010). Each will serve different roles in establishing and maintaining trusting relationships and flows of communication within networks of people (Urry, 2003). Arnfalk and Kogg (2003) appropriately examine through case studies how to develop means to encourage and assist businesses and individuals in choosing the right medium for a given meeting. This could well hold the prospect for the overall removal of some 'unnecessary' face to face meetings either because a virtual alternative is more appropriate or because certain meetings were not necessary at all (entirely or in terms of certain individuals attending).

As the capabilities of alternatives to face to face meetings continue to improve we should remain open to some prospect of a further downwards pressure on face to face meetings that involve travel. This may arise in part through changes to the social norms and expectations that frame a number of the considerations in Table 1. It may also arise through necessity - there are times such as the closure of European air space in the face of the ash cloud where business travel is disrupted (Jain and Guiver, 2012); there may also be effects from rising energy prices or changes to national or international transport policy. 
Multitasking can be defined as the simultaneous conduct of two or more activities during a given time period (Kenyon and Lyons, 2007). For business meetings then multitasking can occur in relation to travel to/from the meeting and within the time period associated with the meeting itself. On the basis that travel itself can be assumed to be a time use then any other activities engaged in during travel (as discussed earlier) can be considered multitasking and the availability of mobile technologies potentially supports and enriches this. Meanwhile ICTS are also beginning to 'invade' meetings themselves. With the increasing availability of wireless or mobile Internet individuals can make use of mobile devices to not only work on documents during a meeting but also to communicate with remote colleagues. Perhaps ironically, individuals can appear to have travelled to a face to face encounter only then to use ICTs to participate in non face to face encounters with others within the same time frame. What individuals are able to do however in this way is to identify parts of a meeting when the value in their being co-present and participating is diminished or marginal and avoid the opportunity cost to some extent by temporarily switching to other activities. This is an evolving phenomenon and not always well received. As the proportion of individuals engaged in this form of multitasking increases within a meeting the more the meeting itself may lose its sense of purpose. Perhaps the phenomenon will evolve to the point where it becomes more evident that some meetings were, therefore, marginal all along in their value and should not take place while other meetings will enforce an etiquette of such multitasking not being engaged in.

\section{An autobiographical insight}

As the reader will be aware, much of the content of this paper (and observations within existing literature) points towards the need for further empirical insights and indeed the merits of a grounded theory approach through qualitative research to develop a richer 
understanding of the influences and social practices surrounding meetings. What the paper does highlight is the challenge of how one captures and codes appropriately the attitudes towards and the behaviour of meeting attendance in such a way as to elicit helpful insights. It seems clear that 'meetingness' is evolving as business and social networks respond to the information age and globalisation as well as to economic and environmental circumstances and concerns. If we are to begin assessing how business travel might change in future (or how it might be changed through policy intervention or business practices) then methodological approaches are needed to elicit further insights into present day practices.

In this section of the paper, I offer one autobiographical example of how one might examine behaviour and attitudes. The example concerns a meeting cluster (see Figure 2) which occurred in London.

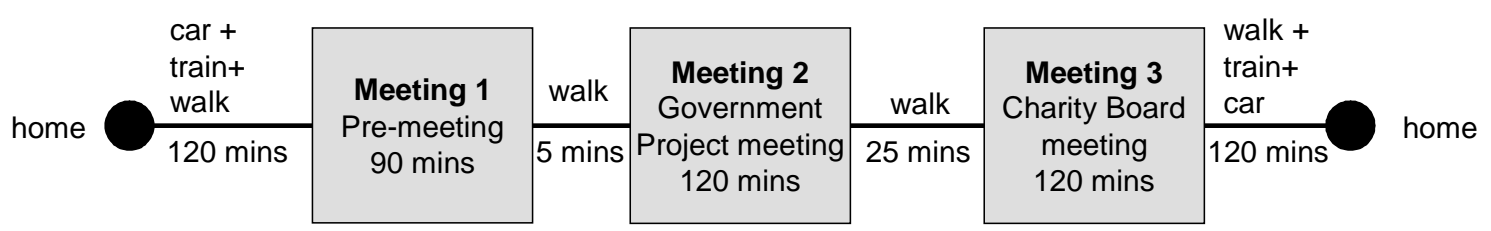

Figure 2. Timeline of autobiographical example of meeting cluster

The original anchor meeting (3) was a quarterly Board meeting at 5pm whose location was dictated by the Charity HQ - Board members were still getting to know one another. By itself this would have involved a travel-time ratio of 0.67 (more time travelling than meeting). The Government was requiring a project meeting at its premises sometime in the same month and I was afforded the flexibility to indicate the best date and time and hence used the anchor meeting as a reference point. This Government meeting (2) gave rise to the need for a pre-meeting over lunch (1). Both meetings involved people who were known to each other. As a result of the additional meetings the travel-time ratio was reduced to 0.45 (more time meeting than travelling). The anchor meeting was less essential to attend than meeting 2. Meetings 2 and 3 were confined to only covering the formal business concerned (i.e. no 
overspills into sociability) and could have been substituted for by teleconferencing ( 2 more so than 3) but in both cases the gesture of co-presence was significant if not essential.

In summary the meeting cluster rendered the participation in all meetings more 'cost' effective but a cultural shift in norms could have afforded the opportunity for all these meetings to have avoided being conducted in a co-present format. My ex-post assessment was as follows:

- meetings 1 and 2 were both productive and a face to face format was worthwhile given the significance of the brainstorming and decision making involved;

- meeting 3 involved only a marginal contribution from myself and while it achieved a goal of information exchange (which could have been done by other means) benefits of attendance were status and recognition alongside a business norm expecting attendance;

- to only have attended meeting 3 would have constituted an opportunity cost but the meeting cluster overall was a worthwhile time investment; and

- travel time to the meetings was fully used for preparation while the travel time on the return journey I treated as non-work time.

This example only scratches the surface of the richness of insight that would be possible in closely examining empirical evidence of how individuals engage in meetings and with what effect. There is still much to learn about how individuals judge the extent to which participation in meetings is worthwhile and the efficacy of different media. Comparison of expost judgement of benefits and disbenefits of attendance with those ex-ante could provide important clues to how to develop better strategies to plan and perhaps rationalise meeting attendance. There is a growing aptitude for and incidence of mixed-method approaches in both transport studies and mobilities research (e.g. Büscher et al (2011)) and further examination of business travel and meetings would benefit from this. 
This paper has sought to begin to 'unpack' the richness of factors and aspects that lie beneath the rather superficial aggregated data regarding business travel captured in national surveys. By highlighting the economic significance afforded to business travel it is argued that the paucity of deeper empirical insight into the nature of business travel and indeed how it is changing over time is rather remarkable. The principal interest of central government, at least in the UK, appears to have been to reduce the burden of business travel on the economy by speeding up journeys to and from meetings through investment in infrastructure and services. Less attention has been given to how the burden might either be: lightened through greater attention to improving the productivity of the travel time involved; or reduced through a reduction in the proportion of business meetings that are required to take place face to face. Given the added impetus to economic considerations that the challenges of carbon reduction have introduced it would seem of added importance to now call for greater attention to be given to understanding the social practices that comprise the construction of business encounters and their associated travel. This would allow a more scrutinising assessment to be made of the extent to which face to face meetings are necessary or 'travel dependent'.

Goodwin (1995) highlighted that in terms of car dependence, a distribution of car trips exists according to the level of necessity for car use - he posited that notionally $20 \%$ of all trips by car unavoidably need to be made by car in terms of the absence of viable alternatives; meanwhile at the other end of the distribution another $20 \%$ of all trips by car arguably have entirely credible alternatives available such that use of car could be avoided. His assertion was that in terms of transport policy it was likely to be more effective, rather than to have blanket policies targeting all car use, to have policies that aimed to 'nibble away' at the $20 \%$ of trips that are not as firmly rooted in a dependence on the car. In later work, Handy et al (2005) have put forward the notion of 'excess driving' and set out a 
conceptual framework that may in fact lend itself to addressing the question of excess business travel or more specifically excess briefcase travel. Handy et al define excess driving as "driving beyond that required for household maintenance given choices about residential location, job location, and activity participation". They go on to note that "[t]he required level of driving can be defined more specifically as the minimum number of trips using the shortest routes to the closest destinations possible and using modes other than the car as often as possible. Excess driving is then defined as driving above and beyond the required level and can be generated by the choice of longer routes, farther destinations, greater use of the car, and more frequent trips than the minimum required." They acknowledge that such minimum requirements can be difficult to define. Handy et al point to a number of reasons for excess driving: value of driving itself; value of activities while driving; variety seeking; habit; poor planning; misperceptions; and lack of information.

I would tentatively suggest that excess briefcase travel could be defined as follows: travel derived from engagement in business encounters involving co-presence where physical presence could have been reasonably substituted for by other means of engagement in the encounter or no encounter needing to take place. Reasons for excess briefcase travel would include those already set out in Table 1. In such a definition the challenge becomes one of how to unpack and interpret what constitutes reasonable substitution - indeed, a further challenge is that this is likely to be changing over time with social and technological change unfolding. In turn the policy challenge and indeed the challenge for employers and employees in relation to business encounters becomes one of how to bring about more judicious decision making about whether, when and how meetings are organised and conducted such that excess briefcase travel is avoided or reduced. I would venture to assert that perhaps the notional figure of $20 \%$ referred to by Goodwin might also apply in this case. 
Anderson, T., Christophersen, O., Pickering, K., Southwood, H. and Tipping, S. (2009). National Travel Survey 2008 Technical Report. Prepared for the Department for Transport, July.

Arnfalk, P. and Kogg, B. (2003). Service transformation - managing a shift from business travel to virtual meetings. Journal of Cleaner Production, 11, 859-872.

Beaverstock, J.V., Derudder, B., Faulconbridge, J.R. and Witlox, F. (2010). International Business Travel and the Global Economy: Setting the Context. In: Beaverstock, J.V., Derudder, B., Faulconbridge, J.R. and Witlox, F. (Eds.), International Business Travel in the Global Economy, Farnham, Ashgate, 1-7.

Bergman, A. and Gustafson, P. (2008). Travel, availability and work-life balance. In Hislop, D. (Ed). Mobility and Technology in the Workplace, Routledge, Chapter 14, 192-208.

Büscher, M., Urry, J. and Witchger, K. (Eds.) (2011). Mobile Methods. Routledge.

Cairns, S., Sloman, L., Newson, C., Anable, J., Kirkbridge, A. and Goodwin, P. (2004), Smarter Choices - Changing the Way We Travel. Department for Transport, London, July.

Chorus, G. C., Molin, E. J. E. and Van Wee, B. (2006). Use and effects of Advanced Traveller Information Services (ATIS): a review of the literature. Transport Reviews, 26, 127-149.

Denstadli, J.M., Julsrud, T.E. and Hjorthol, R.J. (2012). Videoconferencing as a Mode of Communication: A Comparative Study of the Use of Videoconferencing and Face-toFace Meetings. Journal of Business and Technical Communication, 26 (1) 65-91.

DfT (2011). Values of Time and Operating Costs. TAG Unit 3.5.6. Department for Transport, April, London.

DfT (2012). National Travel Survey: 2011. Statistical Release, Department for Transport, London, December. 
Faulconbridge, J.R. and Beaverstock, J.V. (2008). Geographies of international business travel in the professional service economy. In Hislop, D. (Ed). Mobility and Technology in the Workplace, Routledge, Chapter 7, 87-102.

Faulconbridge, J.R., Beaverstock, J.V., Derudder, B. and Witlox, F. (2009). Corporate Ecologies of Business Travel in Professional Services Firms - Working Towards a Research Agenda. European Urban and Regional Studies, 16 (3), 295-308.

Geels, F.W. and Smit, W.A. (2000). Failed technology futures: pitfalls and lessons from a historical survey. Futures, 32, 867-885.

Goodwin, P. (Ed) (1995). Car dependence - A report for the RAC Foundation for Motoring and the Environment. 1st edn. Transport Studies Unit Oxford: RAC Foundation for Motoring and the Environment.

Gustafson, P. (2006). Work-related travel, gender and family obligations. Work, Employment and Society, 20 (3), 513-530.

Handy, S., Weston, L. and Mokhtarian, P.L. (2005). Driving by choice or necessity? Transportation Research A, 39, 183-203.

Haynes, P. (2010). Information and Communication Technology and International Business Travel: Mobility Allies? Mobilities, 5(4), 547-564.

Holley, D., Jain, J. and Lyons, G. (2008). Understanding Business Travel Time Use and its Place in the Working Day. Time \& Society, 17 (1), 27-46.

Hu, P.S. and Reuscher, T.R. (2004). Summary of Travel Trends - 2001 National Household Travel Survey. Report to the US Department of Transportation, December.

Ivancevich, J.M., Konopaske, R. and Defrank, R.S. (2003). Business travel stress: A model, propositions and managerial implications. Work \& Stress: An International Journal of Work, Health \& Organisations, 17 (2), 138-157.

Guiver, J. and Jain, J. (2011) Grounded: Impacts of and insights from the volcanic ash cloud disruption. Mobilities, 6 (1), 41-55.

Jain, J. and Lyons, G. (2008). The gift of travel time. Journal of Transport Geography, 16, 81-89. 
Kenyon, S. and Lyons, G. (2007). Introducing multitasking to the study of travel and ICT: examining its extent and assessing its potential importance. Transportation Research A, 41, 161-175.

Loch, C.H., Tyler, J.R. and Lukose, R. (2003). Conversational Structure in Email and Faceto-face Communication. Hewlett Packard Labs, Palo Alto, October.

Lyons, G. and Urry, J. (2005). Travel time use in the information age. Transportation Research A, 39, 257-276.

Lyons, G., Avineri, E. and Farag, S. (2008a). Assessing the demand for travel information: do we really want to know? Proc. European Transport Conference.

Lyons, G., Holley, D. and Jain, J. (2008b). The Business of Train Travel: A Matter of Time Use. In Hislop, D. (Ed). Mobile Work/Technology: Changing Patterns of Spatial Mobility and Mobile Technology Use in Work, Routledge, Chapter 6, 74-86.

Lyons, G., Jain, J. and Holley, D. (2007). The use of travel time by rail passengers in Great Britain. Transportation Research A, 41, 107-120.

Lyons, G., Jain, J., Susilo, Y. and Atkins, S. (2012). Comparing rail passengers' travel time use in Great Britain between 2004 and 2010. Mobilities.

Mackie, P.J., Wardman, M., Fowkes A.S., Whelan, G., Nellthorp, J. and Bates J. (2003). Value of Travel Time Savings in the UK. Report to the Department for Transport, January.

McNeil, D. (2009). The airport hotel as business space. Geografiska Annaler: Series B, Human Geography, 91 (3), 219-228.

Middleton, C.A. (2008). Do mobile technologies enable work-life balance? Dual perspectives on BlackBerry usage for supplemental work. In Hislop, D. (Ed). Mobility and Technology in the Workplace, Routledge, Chapter 15, 209-224.

Mokhtarian, P.L. and Salomon, I. (2001). How derived is the demand for travel? Some conceptual and measurement considerations. Transportation Research A, 35, 695-719. Romano, N.C. and Nunamaker, J.F. (2001). Meeting Analysis: Findings from Research and Practice. Proc. $34^{\text {th }}$ Hawaii International Conference on System Sciences. 
Schwanen, T. and Dijst, M. (2002). Travel-time ratios for visits to the workplace: the relationship between commuting time and work duration. Transportation Research $A$, 36, 573-592.

Storper, M. and Venables, A.J. (2004). Buzz: face-to-face contact and the urban economy. Journal of Economic Geography, 4 (4), 351-370.

Stradling, S.G. (2004). Changing individual travel behaviour: from policy to perceived behavioural control. Proc. $3^{\text {rd }}$ International Conference of Traffic and Transport Psychology, Nottingham, 5-9 September.

Todd, P.M. (2007). How much information do we need? European Journal of Operational Research, 177, 1317-1332.

Urry, J. (2003). Social networks, travel and talk. British Journal of Sociology, 54 (2), 155-175.

Watts, L. (2008). The Art and Craft of Train Travel. Journal of Social and Cultural Geography, 9 (6), 711-726. 